\title{
Simulated moving bed chromatography separation of galacto-oligosaccharides
}

\author{
Łukasz Wiśniewski, Monika Antošová, Milan Polakovič \\ Department of Chemical and Biochemical Engineering, \\ Institute of Chemical and Environmental Engineering, \\ Faculty of Chemical and Food Technology, Slovak University of Technology, \\ Radlinského 9, 81237 Bratislava, Slovakia \\ milan.polakovic@stuba.sk
}

\begin{abstract}
A mixture of galacto-oligosaccharides and non-prebiotic sugars was separated using a simulated moving bed unit equipped with eight packed-bed columns of a cation exchanger. In order to determine operating conditions using triangle theory, equilibrium adsorption isotherms of galactose, glucose, lactose, tri- and tetragalacto-oligosaccharide were measured. It was found that each column of the SMB unit had a maximum separation efficiency of more than 3000 theoretical plates. The achieved purity of galacto-oligosaccharides in the raffinate stream was $99.9 \%$, similarly $99.9 \%$ of extract was formed by non-prebiotic sugars lactose, glucose and galactose. The productivities in the raffinate and extract, were $0.6 \mathrm{~g} /(\mathrm{L} \mathrm{h})$ and $2.4 \mathrm{~g} /(\mathrm{L} \mathrm{h})$, respectively.
\end{abstract}

Keywords: simulated moving bed chromatography, galacto-oligosaccharides, adsorption isotherm

\section{Introduction}

Galacto-oligosaccharides (GOS) are a class of low caloric, non-cariogenic carbohydrates considered as prebiotics. Large scale production of GOS uses $\beta$-galactosidase as biocatalyst and lactose as a substrate for transgalactosylation reaction (Park and $\mathrm{Oh}$ 2010). It is specific for this process that unreacted lactose as well as by-products, glucose and galactose, form a significant fraction of total saccharides in a post-reaction mixture. The presence of glucose and lactose deteriorates the quality of final value-added product. It also delimits the range of potential consumers. GOS separation from the mentioned low-molecular weight saccharides is a downstream process of economic importance.

Simulated moving bed (SMB) chromatography is a counter-current continuous chromatography technique introduced in 1961 by Broughton and Gerhold as a practical implementation of true moving bed (TMB) concept (Broughton and Gerhold 1961). The first applications of SMB originated in petrochemical industry but it has also found widespread applications in numerous binary separation of enantiomers, active pharmaceutical intermediates and saccharides (Rajendran et al. 2009). Fructose/glucose separation, desugarization of molasses and enrichment of HFCS in fructose belong to the most important implementations of $\mathrm{SMB}$ in sweeteners industry (Azevedo and Rodrigues 2001). Cation exchange resins are typically utilized as adsorbents since they proved their great potential in the separation of carbohydrates over last decades (Chilamkurthi et al. 2012; Nobre et al., Suvarov and De Weireld 2013).
The principle of SMB process can be explained by the mentioned TMB concept which assumes a true counter-current movement of solid and liquid phases (Fig. 1). A hypothetical column is divided into four zones where each zone plays a particular role. Two middle zones are the true separation sections while the boundary zones serve for complete regeneration of solid and liquid phases, respectively. Feed mixture is continuously introduced into the middle of the TMB unit while the eluent and regenerated solid phase enter the bed on the opposite ends. The less retained compounds from the feed move with the mobile phase and are collected at the raffinate port while the more retained compounds move with the solid bed and are recovered at the extract port. In a real SMB process, the solid phase bed does not move but several fixed-bed columns are connected in series and synchronous shifts of inlets and outlets are used. After several cycles, the SMB unit achieves a periodic steady state, i.e. outlet concentrations vary during the cycle around an average value which is constant throughout subsequent cycles.

There are several approaches in the SMB modelling with different simplification levels that lead to the parameter determination and prediction of internal concentration profiles. The simplest way for the determination of operating parameters, i.e. flow rates in each zone and switch time, is the so called triangle theory which is based on adsorption equilibrium. It introduces dimensionless number $m_{j}$ that defines a liquid to solid flow rates ratio in the zone $j$. Imposing constraints on the rates of movement of the separated species, a triangle-shape region of complete separation can be delimited. 
Separation of galacto-oligosaccharides from nonprebiotic saccharides present in their mixture was a subject of this work. A simple approach called "cut through a chromatogram" was proposed in order to collect all GOS in the raffinate and lactose, glucose and galactose in the extract. The triangle theory was used for the first setting of operating parameters. No dynamic simulations were performed. Adsorption equilibrium data of all major components of the mixture were measured using simple batch chromatography. The column efficiency was also assessed to keep the recommendations for application of triangle theory.

\section{Materials and methods}

\section{Materials}

Glucose, lactose and galactose were from SigmaAldrich (St. Louis, USA). Vivinal GOS syrup was kindly provided by FrieslandCampina (Netherlands). It contained $75 \%$ of dry matter. The content of saccharides in the dry matter was as follows: galacto-oligosaccharides - $59 \%$, lactose - $21 \%$, glucose $-19 \%$, and galactose $1 \%$. The term water used throughout this paper means redistilled and filtered water through nitrate cellulose filter (Sartorius, Germany).

\section{Determination of distribution coefficients}

The distribution coefficients $K_{i}$ of the linear isotherm for individual saccharides were determined by the pulse method. The injection volume was $100 \mu \mathrm{L}$. The feed concentration values of particular saccharides were varied in order to cover the conditions occurring during the separation. The feed concentration ranges used were as follows:

- galactose (Gal): 3.125-25 g/L,

- glucose (Glc): 10-75 g/L,

- lactose (Lac): 25-120 g/L,

- galactotriose (GOS3): 10-50 g/L.

The retention time, $t_{R}$, for each pulse experiment was obtained from the first statistical moment (Eq. (1)):

$$
t_{R}=\frac{\int_{0}^{\infty} t C(t) \mathrm{d} t}{\int_{0}^{\infty} C(t)}
$$

The retention times of all saccharides were independent of feed concentration. The distribution coefficient, $K_{i}$, of the linear isotherm was proportional to the retention times and calculated from the following equation:

$$
t_{R}=\frac{\varepsilon V_{C}}{Q}\left(1+\frac{1-\varepsilon}{\varepsilon} K_{i}\right)
$$

where $\varepsilon$ is the interstitial porosity, $K_{i}$ is the distribution coefficient of the component $i, V_{C}$ is the bed volume and $Q$ is the volumetric flow rate.

\section{Determination of column efficiency}

The column efficiency was characterized by the number of theoretical plates, $N_{i}$, which was obtained from an elution profile of pulse injection experiment. The values of $N_{i}$ for lactose and GOS3 were calculated from the retention time, $t_{R, i}$ and peak width, w, using the following equation:

$$
N_{i}=\left(\frac{t_{R, i}}{w}\right)^{2}
$$

The value of $N_{i}$ was determined for several volumetric flow rates in the range of $0.1-4.0 \mathrm{~mL} / \mathrm{min}$. The height equivalent to the theoretical plate (HETP) was then calculated from the equation:

$$
H E T P=\frac{L}{N_{i}}
$$

where $L$ is the column bed length.

\section{SMB unit and experiments description}

SMB experiments were performed using the simulated moving bed chromatography system CSEP C916 (Knauer, Berlin, Germany). This unit is equipped with a 64-port multifunctional rotary valve. Eight columns in the configuration $2-2-$ $2-2$ (two columns per each section) were connected in series and mounted to a rotating carousel. The rotary valve and columns are built in a housing box thermostat that enables to keep a constant temperature up to $60^{\circ} \mathrm{C}$. The unit was equipped with Vertex Eurokat Na $(250 \times 20 \mathrm{~mm})$ columns (Knauer). The flow rates in the unit were set by four independently controlled HPLC pumps from Knauer. The eluent flow rate and the flow rates in the zones II and IV (Fig. 1) were provided by Smartline 100 pumps (Knauer). The feed solution was pumped via a Smartline K1050 HPLC pump (Knauer). The feed and eluent streams were continuously degassed using the Smartline Manager 5050 (Knauer). The volumetric flow rates in the zones II and IV were regulated by the mini CORI-FLOW mass flow controllers (Bronkhorst, Netherlands). The entire system was connected to a PC and was controlled by the ChromGate software.

SMB chromatography experiments were performed at the constant temperature of $60^{\circ} \mathrm{C}$. Water was used as eluent. Vivinal GOS syrup, diluted with water to the total saccharide concentration of $200 \mathrm{~g} / \mathrm{L}$, was used as feed solution. Samples collected at the raffinate and extract ports were analyzed by the HPLC method described below in Analytical method. The unit was fed with the feed and eluent until a periodic 


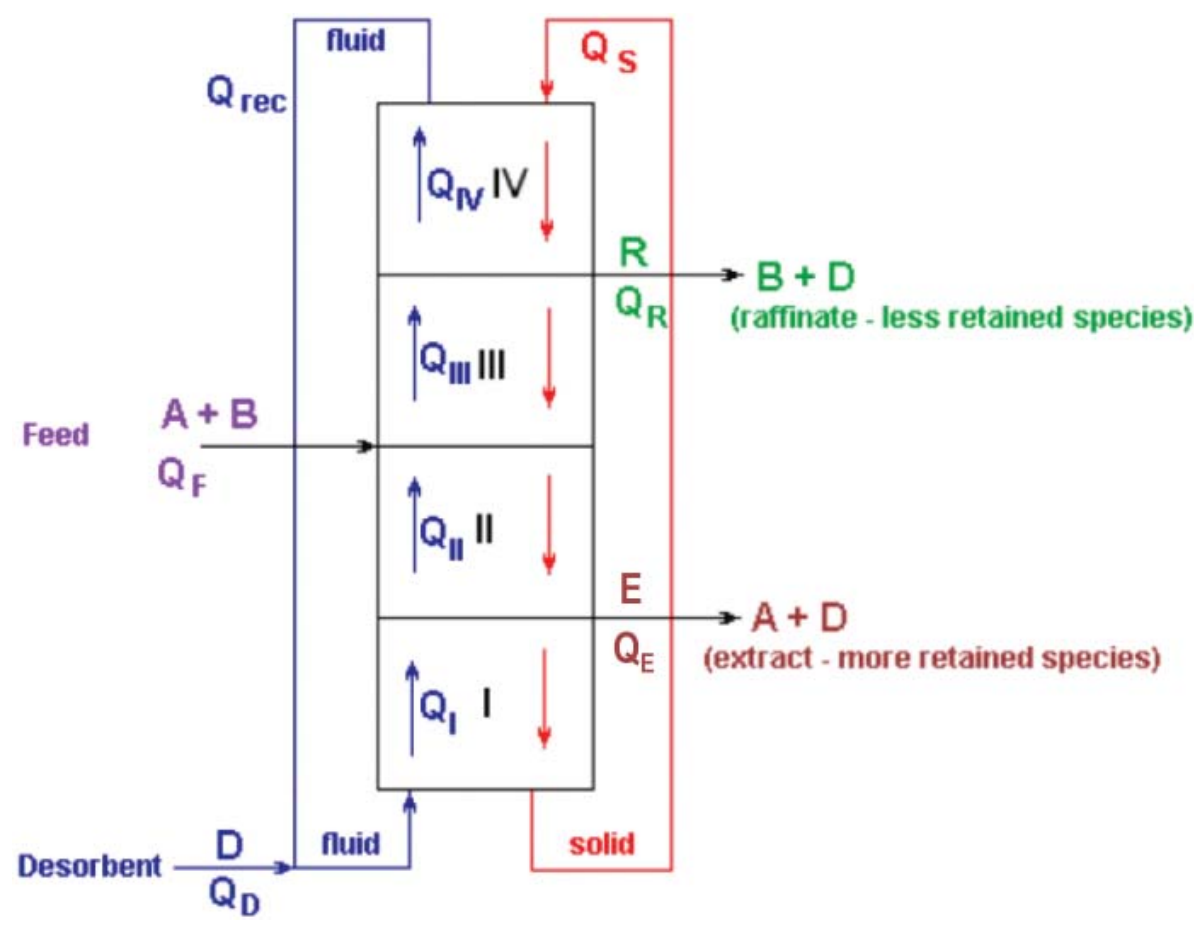

Fig. 1. True moving bed chromatography principle. Roman numerals denote four zones of TMB chromatography. Other symbols: A and B more and less retained species,

$\mathrm{D}$ - desorbent/eluent, $\mathrm{E}$ - extract, $\mathrm{R}$ - raffinate, $\mathrm{Q}$ - flow rate, and subscripts: rec - recycling, F - feed, $\mathrm{S}$ - solid phase.

steady state, recognized by constant concentration profiles of raffinate and extract, was achieved.

\section{Determination of operating conditions}

The operation of the SMB unit required to set five parameters: the flow rates in each zone and switch time (tact time) determining the velocity of the simulated movement of solid phase. Those parameters were determined by the short-cut method based on the equilibrium theory and equivalence of the TMB and SMB processes. The equivalence between the dimensionless ratios of the flow rates can be expressed as follows:

$$
m_{j}=\left.\frac{Q_{j}}{Q_{S}}\right|^{T M B} m_{j}=\left.\frac{Q_{j} t^{*}-\varepsilon V_{C}}{(1-\varepsilon) V_{C}}\right|^{S M B}
$$

where $Q_{j}$ is the volumetric flow rate in zone $j, Q_{S}$ is the solid phase flow rate, $t^{*}$ is the switch time.

The following conditions must be fulfilled to achieve complete separation between GOS3 and lactose in sections II and III:

$$
K_{G O S 3}<\frac{Q_{I I}}{Q_{S}}, \frac{Q_{I I I}}{Q_{S}}<K_{L a c}
$$

where

$$
\begin{aligned}
Q_{i} & =Q_{j}+\frac{\varepsilon}{1-\varepsilon} Q_{S} \\
Q_{s} & =\frac{(1-\varepsilon) V_{C}}{t^{*}}
\end{aligned}
$$

In order to determine the values of velocity ratios in the separation zones II and III, $m_{I I}$ and $m_{I I I}$, the distribution coefficients of lactose and GOS3 were used as the key components. The complete regeneration of zones I and IV was defined for the most and the least adsorbed species present in the mixture. In the case of zone I, $K_{\text {Gal }}$ was used as the compound with the highest affinity to the stationary phase, to fulfil the following inequality:

$$
\frac{Q_{I}}{Q_{S}}>K_{G a l}
$$

The conditions for zone IV were determined in a less straightforward way. Higher GOS (degree of polymerization higher than 4) were poorly separated and distribution coefficient for the largest (the least adsorbed) galacto-oligosaccharide was unavailable. The distribution coefficient of tetragalacto-oligosaccharide (GOS4) was therefore taken to specify the purity of the desorbent (water) in zone IV:

$$
\frac{Q_{I V}}{Q_{S}}>K_{G O S 4}
$$

The flow rate in zone I, which was the highest one, was fixed beforehand. The remaining zone flow rates resulted from this fixed value and correlations defining complete separation by the triangle theory. The values of $Q_{\text {Feed }}$ and $Q_{\text {Eluent }}$ were then calculated from the material balances. 


\section{Analytical method}

Raffinate and extract samples were collected at fixed time intervals and analyzed by HPLC. Diluted samples in water as a mobile phase were injected into the analytical column REZEX RSO-Oligosaccharide $\left(\mathrm{Ag}^{+}\right.$form, $200 \times 10 \mathrm{~mm}$, Phenomenex, USA) by an automatic injector Gilson 234 (France). A differential refractometer (Knauer) was used as a detector. The analyses were carried out at $40{ }^{\circ} \mathrm{C}$ when the temperature was maintained by a thermostat from Thermotechnic Products (Austria). The applied flow rate of $0.3 \mathrm{~mL} / \mathrm{min}$ was provided by the HPLC pump K1001 (Knauer).

\section{Results and discussion}

The characterization of a column from the SMB column series is an essential step that must be carefully performed before a SMB separation process will be designed. Adsorption equilibrium isotherms, porosities and column efficiencies belong to the crucial operation parameters to be determined. Following our previous experience, a linear shape of equilibrium isotherms was assumed. The distribution coefficients, $K_{i}$, of single-component equilibrium adsorption isotherms were obtained by preparative pulse experiments for GOS3, lactose, glucose, and galactose. Due to the missing analytical standard of GOS4, its distribution coefficient was estimated from the retention time for a pulse injection of Vivinal GOS, where all other peaks on the chromatogram obtained were well resolved. The values of all distribution coefficients are summarized in Table 1. It is worth mentioning that the retention times obtained by the pulse injections of single-component solutions were virtually identical with those obtained for the pulse injection of the multicomponent GOS mixture.

Table 1 clearly presents a well known size-exclusion effect as a governing phenomenon in the separation of saccharides using cation exchangers. All obtained values of $K_{i}$ are relatively low. The accessible pore volume for GOS4 is very low. It can be assumed that higher galacto-oligosaccharides are fully excluded from the pores of this adsorbent. The considerable difference in the distribution coefficients of glucose and galactose shows that size-exclusion is not the sole mechanism involved in the partitioning of the saccharides. A different orientation of $-\mathrm{OH}$ groups at carbon $\mathrm{C} \# 4$ is the only structural difference between these two saccharides. The difference in their adsorption behaviour lies in the complex nature of ligand exchange chromatography that combines ion-exclusion, complexation effect, cation hydration, hydrogen bonds, and manifest here its influence (Gramblička and Polakovič 2007; Vaňková et al. 2010).

The obtained values of $N_{i}$ (Eq. (3)) for both lactose and GOS3 ranged from about 1000 up to 3200 depending on the flow rate. The corresponding

Tab. 1. Values of distribution coefficients, $K_{i}$, of major components of Vivinal GOS mixture.

\begin{tabular}{cccccc}
\hline & Galactose & Glucose & Lactose & GOS3 & GOS4 \\
\hline $\mathrm{K}[-]$ & 0.278 & 0.227 & 0.117 & 0.061 & 0.030 \\
\hline
\end{tabular}

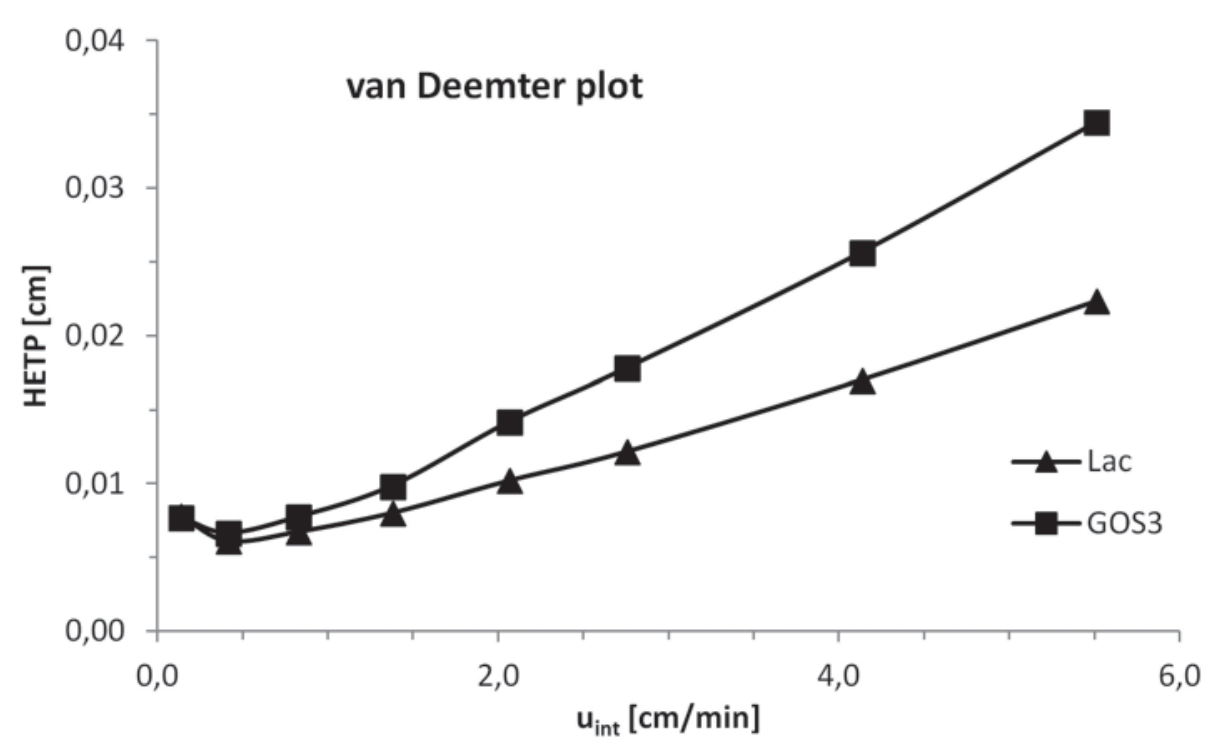

Fig. 2. van Deemter plots for GOS3 and lactose as a function of interstitial velocity; the flow rate range of $0.1-4.0 \mathrm{~mL} / \mathrm{min}$. 
HETP values were calculated from Eq. (4) and they are displayed in the form of van Deemter plots in Fig. 2.

In the typical preparative chromatography, mobile phase velocities used are from the linear part of van Deemter plot (Juza 1999). Table 2 shows that the operation flow rates in the SMB chromatography separation of GOS were $2-4 \mathrm{~mL} / \mathrm{min}$. These values correspond to the right half of the van Deemter plots depicted in Fig. 2. It is worth mentioning that $N_{L a c}$ at the maximal tested velocity of about $5.5 \mathrm{~cm} / \mathrm{min}$ was still higher than 1000 which is an excellent value of the separation efficiency. Table 2 presents the values of operating parameters calculated according to the triangle theory and TMB approach using the equilibrium data from Table 2.

Tab. 2. Operating parameters used during the SMB experiment

\begin{tabular}{lc}
\hline Parameter & Value \\
\hline $\mathrm{Q}_{\mathrm{I}}[\mathrm{mL} / \mathrm{min}]$ & 4.00 \\
$\mathrm{Q}_{\mathrm{II}}[\mathrm{mL} / \mathrm{min}]$ & 2.73 \\
$\mathrm{Q}_{\text {III }}[\mathrm{mL} / \mathrm{min}]$ & 2.87 \\
$\mathrm{Q}_{\mathrm{IV}}[\mathrm{mL} / \mathrm{min}]$ & 2.45 \\
$m_{I}[-]$ & 0.25 \\
$m_{I I}[-]$ & 0.07 \\
$m_{I I I}[-]$ & 0.09 \\
$m_{I V}[-]$ & 0.03 \\
$t^{*}[\mathrm{~min}]$ & 8.45 \\
\hline
\end{tabular}

The chosen operating parameters resulted in excellent values of purities of GOS in the raffinate and of remaining saccharides in the extract stream. The raffinate contained only mixture of GOS (99.9\%) while the extract was GOS-free and lactose, glucose and galactose formed $99.9 \%$ of this fraction. This outcome is even more interesting if one realizes that all band broadening effects as well as the dead volumes of the unit were neglected in the design. Moreover, a way, by which the operating parameters were determined, shows that this simple short-cut method is effective tool for preliminary experiments, provided that SMB columns are highly efficient. It should also be noted that small dead volumes of the system, dramatically reduced by the implementation of the multi-way rotary valve, contributed to this result. A straightforward approach of the "cut through the chromatogram" presented here for binary fractionation of a multicomponent mixture by SMB chromatography expands typical application of this technique and proved to be applicable even when target fraction contains more than one compound (Huthmann and Juza 2005).
The obtained productivity values (calculated per unit volume of adsorbent) were lower than it could be expected for an SMB system. The productivity in the extract stream was $1.8 \mathrm{~g} /(\mathrm{L} \mathrm{h})$ and in the raffinate stream, it was $0.6 \mathrm{~g} /(\mathrm{L} \mathrm{h})$. These somewhat lower values were a consequences of the imposed flow rate values in zones I and IV for their complete regeneration to not risk streams contamination by the recycled stream. Moreover, while designing, overall pressure drop in the system was also taken into account in the design of the operating flow rates. This also led to the moderate throughputs. The obtained products dilutions were 8.83 for the extract: and 5.42 for the raffinate. These values are moderate for an SMB system (Vaňková and Polakovič 2012).

A periodic steady state, recognized by the constant mean concentrations of the raffinate and extract, was achieved after four full cycles from the startup. It means that each column passed four times by each zone. It was studied by other authors, that under linear conditions, period from the start-up to periodic steady state does not depend on columns efficiency (Yun et al. 1997).

\section{Acknowledgement}

This work was supported by the Agency of the Ministry of Education, Science, Research and Sport of the Slovak Republic for the Structural Funds of EU under the grant number ITMS 26240220057.

\section{References}

Azevedo DCS, Rodrigues AE (2001) AIChE Journal 47: 2042-2051.

Broughton DB, Gerhold GG, US Patent 2,985,589 (1961).

Chilamkurthi S, Willemsen JH, van der Wielen LAM, Poiesz E, Ottens M (2012) Journal of Chromatography A 1239: 22-34.

Gramblička M, Polakovič M (2007) Journal of Chemical \& Engineering Data 52: 345-350.

Huthmann E, Juza M (2005) Journal of Chromatography A 1092: 24-35.

Juza M (1999) Journal of Chromatography A 865: 35-49.

Nobre C, Suvarov P, De Weireld G (2013) New Biotechnology DOI:10.1016/j.nbt.2013.06.001.

Park AR, Oh DK (2010) Applied Microbiology and Biotechnology 85: 1279-1286.

Rajendran A, Paredes G, Mazzotti M (2009) Journal of Chromatography A 1216: 709-738.

Vaňková K, Polakovič M (2012) Chemical Engineering \& Technology 35: 161-168.

Vaňková K, Gramblička M, Polakovič M (2010) Journal of Chemical \& Engineering Data 55: 405-410.

Yun T, Bensetiti Z, Zhong G, Guiochon G (1997) Journal of Chromatography A: 175-190. 